\title{
Extracellular Optical Recording Configuration for Neuronal Action Potential Detection by using Surface Plasmon Resonance: Preliminary Experiment
}

\author{
Hyoungwon Baac ${ }^{1,3}$, Sang Beom Jun ${ }^{1,2}$, James N. Turner ${ }^{2}$, William Shain ${ }^{2}$, Karen L. Smith ${ }^{2}$, Michael L. Shuler ${ }^{3}$, and \\ Sung June $\mathrm{Kim}^{1}$ \\ ${ }^{1}$ Nano-Bioelectronics and Systems Research Center, Seoul National University, Seoul 151-742, Korea \\ ${ }^{2}$ Wadsworth Center, New York State Department of Health, Albany, NY 12201-0509 USA \\ ${ }^{3}$ School of Chemical and Biomolecular Engineering, Cornell University, Ithaca, NY 14853 USA
}

\begin{abstract}
We propose an extracellular optical recording configuration for neuronal action potential detection by using Surface Plasmon Resonance (SPR). The method does not use fluorescence dyes but still taking advantages of optical recording. As a preliminary experiment, the electrochemical SPR effect without neurons was investigated. This showed that a high resolution SPR setup $\left(10^{-4} \sim 10^{-5}\right.$ angular shift or more) is required to overcome noise and improve SPR signal. Several issues for neural signal recording are addressed to modify SPR optical configuration.
\end{abstract}

Keywords - action potential recording, neuron, surface plasmon resonance

\section{INTRODUCTION}

Multi-channel simultaneous recording of neural action potential is on fundamental demand for both understanding neuron-neuron communication in network level and developing neural engineering devices or sensors [1,2]: for example, neural probes for brain-computer interface and in vitro neural cellbased biosensors. Especially for in vitro extracellular recording, common approaches to multi-channel configuration are to use either microelectrode arrays $[3,4]$ or voltage-sensitive fluorescence dyes [5-7]. Microelectrode techniques have difficulties associated with recording from microelectrode sites in terms of 'electrode impedance' [8]. The measured signal quality is limited by electrical characteristics of electrode site including charge delivery capacity and electrode area. Signal transmission may be disturbed by various mechanisms such as biological and chemical artifacts as well as capacitive coupling by power line. In contrast, the optical recording scheme using voltage-sensitive dyes is free from terms of impedance. Multichannel recording process is realized in a relatively simple way because multiple signal acquisition is performed in photodetector stage far from neuron-substrate interface [9]. Moreover, a site of choice for recording is not restricted by predetermined location. However, the change in membrane potential should be mediated by electro-optical properties of voltage-sensitive organic dyes that limit the signal quality. The use of fluorescence dyes restricts recording duration due to fluorescence decay. Their molecular toxicity may potentially affect physiological environment. Therefore, it would be valuable to develop more efficient way to overcome the problems associated with both microelectrodes and fluorescence dyes while gaining the inherent advantages of these methods.

Surface Plasmon Resonance (SPR) can be induced at the interface between metal and dielectric medium [10]. It is generated mostly by laser beam in forms of collective electron oscillation along the interface. The SPR condition depends on dielectric properties of both metal and dielectric medium. Such properties at the interface can be electrochemically modulated by external electric field application. Electrochemical SPR in aqueous solution has been widely used to study the metalelectrolyte interface [11-12]. Recently, cyclic voltammetry showed that the change in charge density less than hundred $\mu \mathrm{C} / \mathrm{cm}^{2}$ can be detected by resonance angle shift on the order of $\Delta \theta \sim 10^{-3}$ where 0 to -0.3 or 0 to 0.3 voltage steps are applied [12]. The variation of voltage and charge would be smaller during action potential passage of neurons. However, highly sensitive SPR configurations have been steadily developed enabling to measure $\Delta \theta \sim 10^{-5}$ or more [13]. This offers a new opportunity for more sensitive applications.

It is challenging to apply SPR to measure such small neuronal activity as action potentials. Optical recording based on SPR will allow direct detection of electrical signal at metalelectrolyte interface, without use of fluorescence dyes. The signal will not be interfered by other mechanisms during signal transmission since the recording spot is wirelessly addressed by laser beam. Multi-channel simultaneous recording may also be done simply in photodetector stage utilizing photodiode array or CCD apparatus. The imaging application of SPR has already been studied for applications to DNA [14], protein [15], and cell [16]. Here, as preliminary work, we investigate electrochemical modulation of SPR effect without neurons. Issues in recording neural action potential and ways to overcome limitations are discussed with combining the SPR optical configuration.

\section{II . EXPERIMENTAL}

\section{A. Electrochemical SPR Setup}

Fig. 1 illustrates a brief schematic of electrochemical SPR setup. Kretschmann configuration [10] with $632.8 \mathrm{~nm} \mathrm{He}-\mathrm{Ne}$ 


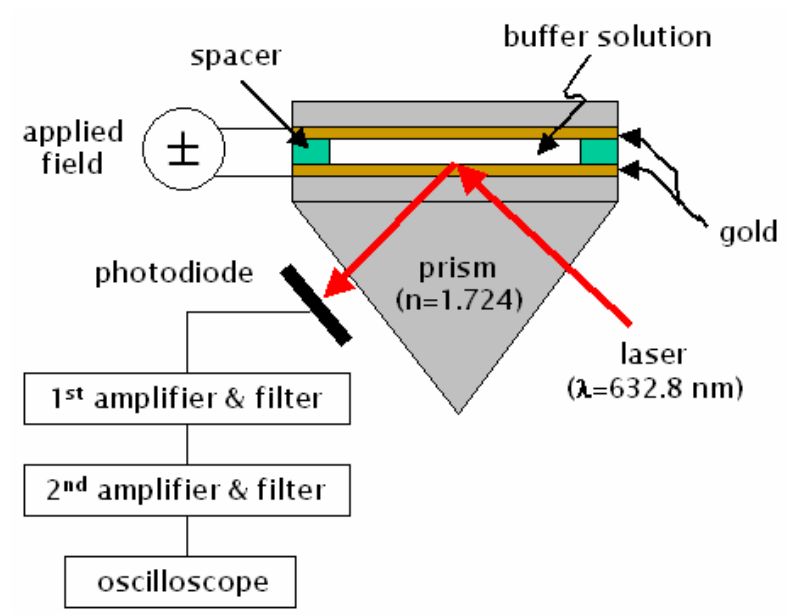

Fig. 1. Schematic view of SPR setup. Prism is mounted on motorized rotation stage. Voltage input is given at $1 \mathrm{kHz}$ by function generator. Signal conditioning is performed through two stages (see text for detail).

laser beam is used to generate SP. High refractive index prism and glass slide (SF-10) are attached by index matching liquid. Titanium layer for adhesion is evaporated over the glass slide by $1 \mathrm{~nm}$ thickness, which is followed by $49 \mathrm{~nm}$ thick gold layer formation. Two gold-coated glass slides are assembled into a sandwich-type sample with $70 \mu \mathrm{m}$ gap which is filled by Phosphate Buffer Solution (PBS) with $\mathrm{pH}=7.4$. The prism is mounted on motorized rotation stage (Newport, SR50PP). Its angular rotation is digitally controlled. Electric function generator (Hewlett Packard, 3314A) is used to apply $1 \mathrm{kHz}$ electric field across the sample. The reflected beam irradiates to silicon photodiode (Hamamatsu, S2387-1010R). The photodiode current output is converted to voltage and amplified by $\times 100,000$ with $6 \mathrm{~dB}$ bandpass filter at $1 \mathrm{kHz}$ cutoff frequency (low and high cutoff points are same) at the first stage (Stanford Research Systems, SR570), and again by $\times 1,000$ with $20 \mathrm{~dB}$ bandpass filter at $0.3-1 \mathrm{kHz}$ (Dagan, Model 2400). Finally, the signals are measured by analog oscilloscope (BK Precision, Model 2160).

\section{B. Electrochemical Modulation Condition}

Fig. 2(a) shows an angular profile of SPR in our setup. Differential reflectance in Fig. 2(b) shows a maximum variation point in reflectance, $\sim 55.7^{\circ}$ (dotted line). Incidence angle of laser beam is fixed to $55.7^{\circ}$ while we modulate the SPR by external electric field. Three different voltages, $50 \mathrm{mV}$, $30 \mathrm{mV}$, and $10 \mathrm{mV}$ (peak to peak, rectangular waveform) are applied with the same frequency, $1 \mathrm{kHz}$.

\section{RESULTS AND DISCUSSION}

\section{A. Electrochemical Modulation of SPR Effect}

SPR signals were electrochemically modulated following the applied field frequency. Fig. 3 shows typical time traces of

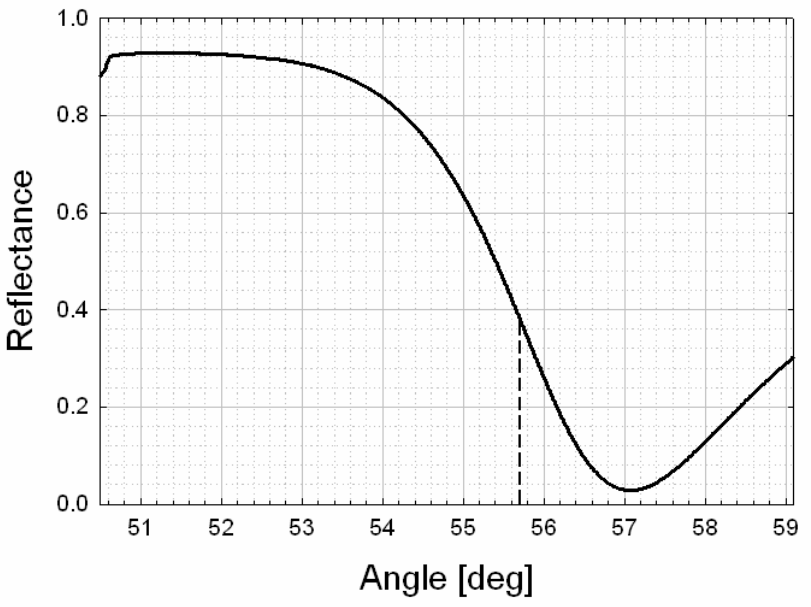

(a)

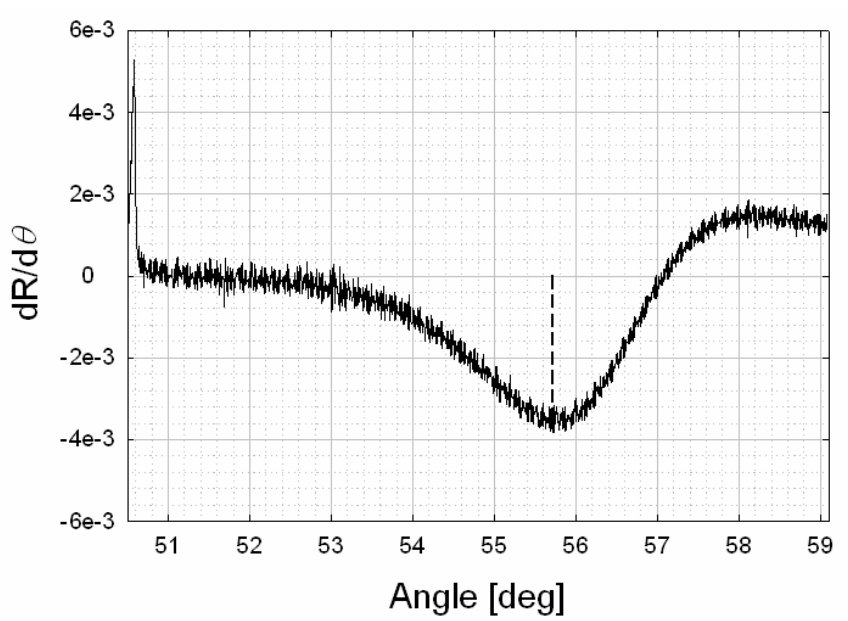

(b)

Fig. 2. SPR curve: (a) Reflectance and (b) Differential reflectance. Differential reflectance is directly calculated from (a).

modulated reflectance (green) for three applied voltages: $50 \mathrm{mV}$, $30 \mathrm{mV}$, and $10 \mathrm{mV}$. Light modulation was distinguishable for $50 \mathrm{mV}$ and $30 \mathrm{mV}$ cases although signal profiles were not fully consistent due to noise. Typically, more than $15 \mathrm{mV}$ of voltage application allowed visual distinction in oscilloscope screen. Signal distortion was severe under $10 \mathrm{mV}$.

We believe that the signal distinction should become clear at least near a few $\mathrm{mV}$ as we consider small action potential spikes observed in common extracellular recording process by microelectrode. The preliminary result in Fig. 3 reveals that the signal quality should be improved to overcome fundamental noises such as laser beam fluctuation, thermal noise in the photodiode, and mechanical vibration probably involved.

\section{B. Issues for better signal quality and neural action potential recording}

The current SPR optical setup allows to measure the resonance angle shift of $\sim 10^{-3}$. For detecting small signals 


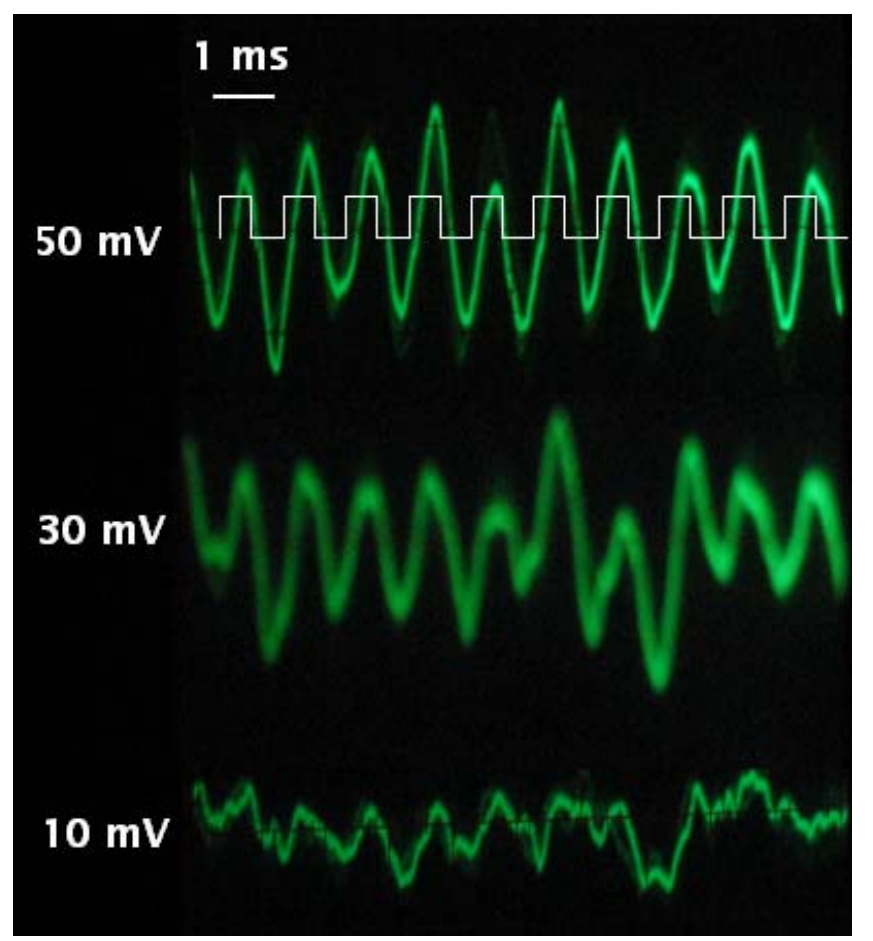

Fig. 3. Electrochemical modulation of SPR reflectance. Green lines are light intensity (arbitrary scale) for three different applied voltages. White rectangular wave is overlapped for guiding the time trace of applied voltage (arbitrary scale). All signal traces are measured by oscilloscope and captured by digital camera (shutter speed $=1 / 1000 \mathrm{sec}$ ). Blurred images are due to the limitation of camera shutter speed.

electrochemically modulated, a highly sensitive and reliable setup is required, which can measure $10^{-4} \sim 10^{-5}$ angular shift. Such sensitivity will be the level endurable against most of fundamental noises. Recently, SPR configurations using multicell photodiodes have been proposed [11-13]. Briefly, such scheme is to acquire two or four signals from multiple elements of photodiodes and compensate each other to discard noises included in signals. This approach enabled to measure highly sensitive signals, $\sim 10^{-5}$ angular shift or more. For neural signal recording, signal conditioning apparatus should be connected with each photodiodes: 4-pole Bessel filters and high gain transimpedance amplifiers for each photodiodes [9]. This means that two or four photodiodes are required for single spot measurement.

Neuronal cell culture on gold substrates will make an adverse environment for SPR configuration in comparison to the case without neurons. Various biological species secreted from neurons will form layers over gold surface in nonuniform way. This may disturb to obtain consistent SPR signals because the SPR is sensitive to surface uniformity associated with light scattering and absorption.

Sensing area is also an issue that should be considered for single neuron analysis. Electrochemical modulation by single neuron needs to be isolated on the sensing spot from other neurons. Incident laser beam can be focused by lens to less than $10 \mu \mathrm{m}$ in diameter fitting into the size of neuronal cell body. The excessive dimension of focused beam, greater than single neuron, may disturb the efficient modulation of SPR signals because it involves the area without the neuron.

\section{SUMMARY}

We propose an extracellular optical recording configuration for neuronal action potential detection by using SPR. The method does not need fluorescence dyes but still has the advantages of optical recording. As a preliminary experiment, the electrochemical SPR effect was investigated without neurons. This showed that high resolution SPR setup is required to overcome noises and improve SPR signal quality. We expect that an advanced SPR configuration using multi-cell photodiodes will be a promising solution capable of detecting $10^{-4} \sim 10^{-5}$ angular shift. Several issues for neural signal recording were also addressed to modify SPR optical configuration.

\section{ACNKOWLEDGMENT}

This work was supported by Korea Science and Engineering Foundation (KOSEF) through Nano Bioelectronics and Systems Research Center (NBS-ERC) in Seoul National University, Nanobiotechnology Center (NBTC), an STC Program of the National Science Foundation (Agreement No. ECS-9876771), and the Cornell Nanoscale Facility (Agreement No. ECS-9731293).

\section{REFERENCES}

[1] W. L. C. Rutten, "Selective electrical interfaces with the nervous system," Annu. Rev. Biomed. Eng. vol. 4, pp. 407-452, 2002.

[2] S. J. Oh, J. K. Song, S. J. Kim, "Neural Interface with a Silicon Neural Probe in the Advancement of Microtechnology," Biotechnol. Bioprocess Eng., vol. 8(4), pp. 252-256, 2003.

[3] C. D. James, A. J. H. Spence, N. M. Dowell-Mesfin, R. J. Hussain, K. L. Smith, H. G. Craighead, M. S. Isaacson, W. Shain, and J. N. Turner, "Extracellular Recordings From Patterned Neuronal Networks Using Planar Microelectrode Arrays," IEEE Transactions on Biomedical Engineering, vol. 51, no. 9, pp. 1640-1648, 2004.

[4] Y. Nam, J. C. Chang, B. C. Wheeler, and G. J. Brewer, "Gold-Coated Microelectrode Array With Thiol Linked Self-Assembled Monolayers for Engineering Neuronal Cultures," IEEE Transactions on Biomedical Engineering, vol. 51(1), pp. 158-165, 2004.

[5] S. Antic and D. Zecevic, "Optical Signals from Neurons with Internally Applied Voltage-Sensitive Dyes," The Journal of Neuroscience, vol. 15(2), pp. 1392-1405, 1995.

[6] S. Antic, G. Major, and D. Zecevic, "Fast Optical Recordings of Membrane Potential Changes From Dendrites of Pyramidal Neurons," J. Neurophysiol., vol. 82, pp. 1615-1621, 1999.

[7] D. A. Dombeck, M. Blanchard-Desce, and W. W. Webb, "Optical Recording of Action Potentials with Second-Harmonic Generation Microscopy," The Journal of Neuroscience, vol. 24(4), pp. 999-1003, 2004.

[8] T. Stieglitz, "Electrode materials for recording and stimulation," in Neuroprosthetics: Theory and Practice, vol. II, K. W. Horch and G. Dhillon, Eds. Singapore: World Scientific Publishing, 2004, pp. 475-516.

[9] J. Y. Wu, L. B. Cohen, and C. X. Falk, "Fast Multisite Optical Measurement of Membrane Potential, with Two Examples," in Fluorescent and Luminescent Probes for Biological Activity, W. T. Mason, Ed. $2^{\text {nd }}$ Ed., London: Academic Press, 1999, pp. 222-237.

[10] H. Raether, Surface Plasmons, Berlin, Heidelberg, New York: Springer Verlag, 1988.

[11] N. J. Tao, S. Boussaad, W. L. Huang, R. A. Arechabaleta, and J. D’Agnese, 
"High resolution surface plasmon resonance spectroscopy," Rev. Sci. Inst., vol. 70(12), pp. 4656-4660, 1999.

[12] J. E. Garland, K. A. Assiongbon, C. M. Pettit, and D. Roy, "Surface plasmon resonance transients at an electrochemical interface: time resolved measurements using a bicell photodiode," Analytica Chimica Acta, vol. 475, pp. 47-58, 2003.

[13] H. Q. Zhang, S. Boussaad, and N. J. Tao, "High-performance differential surface plasmon resonance sensor using quadrant cell photodetector," Rev. Sci. Inst., vol. 74(1), pp. 150-153, 2003.

[14] B. P. Nelson, T. E. Grimsrud, M. R. Liles, R. M. Goodman, and R. M.
Corn, "Surface Plasmon Resonance Imaging Measurements of DNA and RNA Hybridization Adsorption onto DNA Microarrays," Anal. Chem., vol. 73, pp. 1-7, 2001.

[15] E. A. Smith, W. D. Thomas, L. L. Kiessling, and R. M. Corn, "Surface Plasmon Resonance Imaging Studies of Protein-Carbohydrate Interactions," J. Am. Chem. Soc., vol. 125, pp. 6140-6148, 2003.

[16] K.-F. Giebel, C. Bechinger, S. Herminghaus, M. Riedel, P. Leiderer, U. Weiland, and M. Bastmeyer, "Imaging of Cell/Substrate Contacts of Living Cells with Surface Plasmon Resonance Microscopy," Biophysical Journal, vol. 76, pp. 509-516, 1999. 